\title{
Relationship of bone density with serum parathyroid hormone in hemodialysis patients; a single center study
}

\author{
Bita Omidvar ${ }^{1}$, Ali Ghorbani ${ }^{*}$, Mohammad Reza Tamadon ${ }^{3}$, Zahra Sadeghian Broujeni ${ }^{2}$, Mohammad \\ Bahadoram $^{4,5}$, Mehrdad Dargahi ${ }^{6}$
}

\begin{abstract}
Introduction: The existence of a relationship between the level of serum parathyroid hormone (PTH) and bone density can be a warning sign in hemodialysis patients.

Objectives: In the studies conducted so far, the relationship between these two factors has not been specifically investigated. Hence, this study was aimed to exclusively evaluate the levels of serum PTH hormone and bone density.

Patients and Methods: In this cross sectional study, 85 eligible chronic hemodialysis patients aged over 18 years old who underwent hemodialysis a minimum of two times a week for at least 6 months were enrolled in the study. Serum intact PTH, $25 \mathrm{OH}$-vitamin D3, CBC, calcium (Ca), phosphorus (P), alkaline phosphatase (ALP), albumin (Alb), and VBG were measured. Additionally, lumbar radiographic profile and densitometry tests were conducted for patients.

Results: The mean age of patients was 52.9 years (20 to 86 years old). Of them, 43 patients were male (50.58\%). There was a significant correlation between age and the decrease in bone density $(P=0.004)$. There was a significant relationship between different groups of bone density and serum vitamin D3 level $(P<0.05)$. Based on the results of $\mathrm{Z}$ standard, a significant difference between the mean levels of intact PTH in different groups of bone density was seen $(P=0.037)$.

Conclusion: The prevalence of osteopenia and osteoporosis in hemodialysis patients is high. In addition, the decrease in bone density is associated with high levels of PTH. While this association is mainly associated with Z score, it is necessary to investigate the secondary causes of this condition.

Keywords: Osteopenia, Osteoporosis, Hyperparathyroidism, Hemodialysis, Chronic Kidney Disease, End-Stage Renal Disease Please cite this paper as: Omidvar B, Ghorbani A, Tamadon MR, Sadeghian Broujeni Z, Bahadoram M, Dargahi M. Relationship of bone density with serum parathyroid hormone in hemodialysis patients; a single center study. J Parathyr Dis. 2018;6(2):57-63. DOI: 10.15171/jpd.2018.19.

Copyright (c) 2018 The Author(s); Published by Nickan Research Institute. This is an open-access article distributed under the terms of the Creative Commons Attribution License, which permits unrestricted use, distribution, and reproduction in any medium, provided the original work is properly cited.
\end{abstract}

\section{Introduction}

End-stage renal disease (ESRD) is a costly and debilitating disease, which its incidence has decreased in recent decades. In this stage of chronic kidney disease (CKD), the accumulation of toxins which normally are excreted by kidney, leads to uremic syndrome. This condition is a fatal condition unless the toxins and waste materials should be excreted and an electrolyte balance would be achieved by either dialysis or kidney transplant (1).

Over recent decades, the incidence and prevalence of the disease has increased. According to a report by the ESRD Medicare-funded program, the proportion of patients with ESRD increased from around 10000 people in 1973 to 86354 people in 1983 , and later it increased to 27283 people on December 31, 2007. This increase in proportion of patients is due to the increase in the incidence of the disease, increase in the proportion of patients who used alternative treatment methods, and increase in the survival rate of patients with end-stage renal failure (2). The prevalence of this condition, which is known as the fifth stage of CKD in the United States, is equal to $0.2 \%$ of the total population of the United States (3). According to the latest statistics published by the ministry of health and medical education, around 32000 dialysis patients exist in Iran. The number of patients with chronic kidney failure is estimated to be 10 times more than the aforementioned number of dialysis patients (4).

Even after the application of alternative treatment methods, the disease is associated with many complications and problems for the patients. Bone disorders that are caused due to the changes in the parathyroid hormone (PTH) and disturbances in electrolyte and acid and base system of the body are among the most important complications of CKD. CKD not only increases the cost and burden of disease due to additional treatments, but also leads to the disability and debilitation of the patients and have a heavy 
Implication for health policy/practice/research/ medical education

In an investigation on 85 chronic hemodialysis patients aged over 18 years old, we found, the prevalence of osteopenia and osteoporosis in hemodialysis patients is high. In addition, the decrease in bone density is associated with high levels of serum parathormone.

impact on patients' quality of life (1). The prevalence of bone disorders in dialysis patients at the global level is reported to be between $33 \%$ and $67 \%$ (5).

As the patients with osteoporosis and other bone disorders are at risk of different types of bone fractures and other disabilities, during treatment it is tried to prevent such complications, diagnosis the affected people and try to control the problems.

Methods to examine bones are two types: qualitative methods (bone biopsy) and quantitative method (densitometry) (6). Dual x-ray absorptiometry (DXA) is recognized as the best method to assess bone density worldwide. Although bone densitometry is one of the best methods to assess the bone system of the patients, it has some limitations such as its high cost. On the other hand, bone densitometry can detect patients only when some changes have occurred in the bone of patients (6).

It seems that $\mathrm{PTH}$, as an important factor in bone changes, plays an important role in the condition of patients with chronic renal failure (1). PTH is the most important physiological regulator of calcium. PTH acts directly on the bone where it induces bone resorption. In addition, it stimulates calcium absorption and the synthesis of vitamin $\mathrm{D}(1)$.

\section{Objectives}

Given that the level of PTH is checked every six months in dialysis patients and as the kidney failure is a costly disease which imposes a very high burden on the family and health system, and finally given the fact that patients who undergo alternative treatments suffer from many physical and psychological problems, hence in case of findings a clear connection between the level of serum PTH and bone density, the PTH levels can be used as an early alarming sign indicating the need for conducting early bone densitometry and then it will prevent the drastic changes in bones. It can also reduce the risk of severe changes in bone and its subsequent fractures, as well as the negative mental and psychological effects on patients. In case of the presence of a significant relationship between serum PTH levels and bone density, PTH can be used as a warning sign indicating the need for using early densitometry in hemodialysis patients.

The studies which have been conducted so far have assessed the factors which contribute to the reduction of bone density. PTH is one of these contributing factors. However, no study has specifically investigated the relationship between these two items. Considering the above mentioned issues, it seems logical and necessary to find a solution to evaluate patients by a low-cost method.

\section{Patients and Methods}

Study population

In this cross-sectional study, which was conducted on 85 chronic hemodialysis patients aged over 18 years old. The eligible patients who underwent hemodialysis a minimum of two times a week for at least six months were enrolled in the study.

Exclusion criteria were; coronary artery diseases or cerebrovascular accident, orthopedic disorders and chronic lung diseases.

In addition, oral explanations were provided for all patients. The required data collected from patients were recorded in a pre-designed form.

\section{Laboratory assessments}

Fasting samples from venous blood were obtained to measure levels of intact PTH (iPTH), T4, Thyroidstimulating hormone (TSH), follicle-stimulating hormone (FSH), testosterone, $25 \mathrm{OH}$-vitamin D3, CBC, calcium (Ca), phosphorus (P), alkaline phosphatase (ALP), albumin (Alb) and magnesium (Mg) and VBG.

Intact $\mathrm{PTH}, \mathrm{T} 4, \mathrm{TSH}, \mathrm{FSH}$, and testosterone tests were assessed by IRMA method, using Immunotech kit (manufactured in the Czech Republic and an automatic gamma counter 1272 apparatus manufactured in Finland). Serum vitamin D measured by enhanced chemiluminescence (ECL) method, using Rosh kit manufactured in Germany with ELEXSYS ${ }^{\text {тм }}$ apparatus.

Additionally, CBC was tested using Sysmex KX-21N ${ }^{\mathrm{mm}}$ apparatus and the other tests were performed by Hitachi 2009 apparatus and Pars test kits. The VBG was also checked as well. A radiographic lumbar profile was conducted to assess the fractures in all the patients.

\section{Bone density assessment}

The densitometry of all patients was measured using Norland apparatus. All the patients were evaluated for 1 year at Emam hospital in Ahvaz.

\section{Ethical issues}

1) The research followed the tenets of the Declaration of Helsinki. 2) Informed consent was obtained. 3) This study was approved by the Ethics Committee of Ahvaz Jundishapur University of Medical Sciences (Ethical issue \# 295). The goals of the study were explained to participants and all of them accepted to participate and were assured consider the confidentiality of their individual information as well as the voluntary nature of participating in the study.

\section{Statistical analysis}

After collecting the data and entering the data into the database, the statistical analysis was conducted by SPSS 16.0 software and $P$ value less than 0.05 was considered 
as significant. To assess the relationships, the Pearson's correlation test was used and to compare the PTH levels, ANOVA test was conducted. The regression logistic analysis was applied to assess the impact of other variables on bone density. To determine the accuracy of iPTH compared to BMD, sensitivity and specificity were calculated.

\section{Results}

In this study, 85 dialysis patients admitted to Imam Khomeini hospital in Ahvaz who underwent dialysis two times a week for at least six months were studied.

The mean age of the patients was 52.9 years (20-86 years). Of them, 43 patients were male (50.6\%) and 42 patients were female (49.4\%). According to the results of densitometry and based on the T scores of femoral neck, 3 of 85 patients (3.5\%) were normal, 15 patients $(17.6 \%)$ had osteopenia, and 67 patients (78.8\%) had osteoporosis. Based on the results of T scores of total femur, five patients (5.9\%) were normal, 34 patients $(40 \%)$ had osteopenia, and 46 patients $(54.1 \%)$ had osteoporosis. Based on the results of $\mathrm{T}$ score of the spine, 27 patients $(31.76 \%)$ were normal, 49 patients (57.6\%) had osteopenia, and nine patients $(10.6 \%)$ had osteoporosis. Based on the results of Z score of femoral neck, the Z score was more than (-2) in 50 patients $(58.8 \%)$ and was less than $(-2)$ in 32 patients (37.6\%). The $\mathrm{Z}$ score of total femur was more than $(-2)$ in 26 patients $(30.6 \%)$ and less than $(-2)$ in 59 patients (69.4\%). The $\mathrm{Z}$ score of lumbosacral was more than $(-2)$ in 71 patients $(83.5 \%)$ and less than $(-2)$ in 11 patients (12.9\%). Moreover, T scores of total femur for different age groups were as follows; the mean age of the patients who had normal densitometry and T score was 44.6 years, the mean age of patients with osteopenia was 47.64 years, and the mean age of patients with osteoporosis was 57.56 years. There was a significant relationship between age and the level of decreased bone density $(P=0.004)$, thus, the increase in the mean age of patients was associated with decreased level of bone density (Tables 1 and 2).

The mean age of patients with normal $\mathrm{T}$ score of femoral was 40 years, while in patients with osteopenia was 48.33 years and in patients with osteoporosis was 54.56 years. Based on the results of $\mathrm{T}$ score, there was no significant relationship between age and decreased level of femoral neck bone density $(P=0.08)$.

Based on the results of $\mathrm{T}$ score, the mean age of the group with normal lumbosacral was 54.96 years, in patients with osteopenia was 50.51 years, and in patients with osteoporosis was 60.22 years. There was no significant relationship between age and decreased level of bone density $(P=0.117)$.

The mean age of the patients who had a $\mathrm{Z}$ score of total femur more than (-2) and less than (-2) was 47.92 years and 54.49 years, respectively. There was a significant relationship between age and the decreased level of bone density, hence with an increase in age, the bone density decreased $(P=0.013)$. The mean age of the patients who had a $\mathrm{Z}$ score more than $(-2)$ and less than (-2) was 55.78 years and 46.5 years, respectively. Therefore, a significant relationship between age and decreased level of bone density was detected. Therefore, lower age was associated with decreased level of bone density $(P=0.03)$.

The mean age of the patients who had a $\mathrm{Z}$ score of spinal cord less than (-2) and more than (-2) was 53.35 years and 44.45 years, respectively. Hence, a significant relationship between age and decreased level of bone density was found. Likewise, lower age was associated with decreased level of bone density $(P=0.047)$.

Patients were evaluated for the effects of confounding factors (Ca, P, Mg, albumin, ALP, TSH, T4, FSH, testosterone and vitamin D3 levels). No significant correlation between bone density and the above

Table 1. Mean and standard deviation of age and gender distribution in different states of osteoporosis in the studied subjects

\begin{tabular}{|c|c|c|c|c|c|}
\hline & & Normal & Osteopenia & Osteoporosis & Total \\
\hline \multicolumn{6}{|c|}{ Score spine } \\
\hline \multirow{2}{*}{ Age } & Mean \pm SD & $55.3(12.04)$ & $50.43(14.68)$ & $58.37(17.88)$ & $52.82(14.16)$ \\
\hline & No. (\%) & 27 (31.76) & 49 (57.64) & 9 (10.6) & $85(100)$ \\
\hline \multicolumn{6}{|c|}{ T score total } \\
\hline \multirow{2}{*}{ Age } & Mean \pm SD & 46.75 (9.8) & $48.06(2.2)$ & $56.9(2.1)$ & $52.82(14.16)$ \\
\hline & No. (\%) & $5(5.87)$ & $34(40)$ & $46(54.1)$ & 85 (100) \\
\hline \multirow{2}{*}{ Gender } & Male (\%) & $2(2.35)$ & 13 (15.29) & $28(32.94)$ & $43(100)$ \\
\hline & Female (\%) & $3(3.52)$ & $21(24.70)$ & $18(21.17)$ & $42(100)$ \\
\hline
\end{tabular}

In osteoporosis group the mean age has a significant difference $(P<0.05)$.

Table 2. Frequency distribution of the subjects based on the state of osteoporosis in different studies areas of the body

\begin{tabular}{cccccc}
\hline & & \multicolumn{3}{c}{ T score } \\
\cline { 3 - 5 } & & Normal & Osteopenia & Osteoporosis & Total \\
\hline \multirow{2}{*}{ Studied area, No. (\%) } & Neck & $3(3.5)$ & $15(17.6)$ & $67(78.8)$ & $95(100)$ \\
& Spine & $27(31.8)$ & $49(57.6)$ & $9(10.6)$ & $85(100)$ \\
\hline
\end{tabular}

There was no statistically significant difference between the groups. 
mentioned factors was detected. According to the total femur $\mathrm{T}$ scores, the relationship between serum vitamin D3 levels and the changes in bone density was as follows; the level of serum vitamin D3 levels in normal group was $62.03 \mathrm{ng} / \mathrm{mL}$, while in osteopenia group it was $27.25 \mathrm{ng} /$ $\mathrm{mL}$, and in osteoporosis group was $28.70 \mathrm{ng} / \mathrm{mL}$. Overall serum vitamin D3 levels were $29.6 \mathrm{ng} / \mathrm{mL}$. A significant relationship between different groups of bone density and the levels of vitamin D3 was seen. Thus, with a decrease in bone density, the level of vitamin D3 decreased $(P<0.05)$ (Table 3).

The mean level of PTH in groups with different bone density was evaluated using $\mathrm{Z}$ scores. We found serum iPTH level in the group with a $\mathrm{Z}$ score more than $(-2)$ and less than (-2) was $400.79 \rho \mathrm{g} / \mathrm{mL}$ and $598.95 \rho \mathrm{g} / \mathrm{mL}$, respectively, while the overall mean level of $\mathrm{iPTH}$ was $478.1 \mathrm{\rho g} / \mathrm{mL}$. Hence a significant difference between the two groups $(P=0.018)$ was seen. As a result, with an increase in iPTH levels, the bone density decreased. Mean levels of iPTH the group with total femur Z score of more than $(-2)$ and less than $(-2)$ was $350.20 \mathrm{pg} / \mathrm{mL}$ and 531.33 $\rho \mathrm{g} / \mathrm{mL}$, respectively, and the overall mean level of iPTH was $475.92 \mathrm{\rho g} / \mathrm{mL}$. We found a significant difference between the groups while, with a decrease in bone density, the level of iPTH increased $(P=0.037)$.

Accordingly, the frequency of bone density in various states of $\mathrm{iPTH}$ level were classified in the three groups of adynamic (iPTH $<100 \mathrm{gg} / \mathrm{mL}$ ), hyperparathyroidism $(\mathrm{iPTH}>450 \mathrm{\rho g} / \mathrm{mL}$ ) and normal (iPTH $=100-450 \mathrm{\rho g} / \mathrm{mL}$ ). Accordingly, 13 patients (15.3\%) were in the adynamic group, 21 patients $(24.7 \%)$ were in the normal group, and 51 patients $(60 \%)$ were in hyperparathyroidism group.

The frequency distribution of patients based on their total femur T scores and in terms of different levels of iPTH was as follows. In patients with $\mathrm{iPTH}<100 \mathrm{\rho g} / \mathrm{mL}$; two patients were normal, nine patients had osteopenia, and two patients had osteoporosis (total; 13 patients). In patients with iPTH $=100-450 \mathrm{\rho g} / \mathrm{mL}$, one patient was normal, six patients had osteopenia, and 14 patients had osteoporosis (total; 21 patients). In patients with $\mathrm{iPTH}>450 \mathrm{\rho g} / \mathrm{mL}$, two people were normal, 19 patients had osteopenia, and
30 patients had osteoporosis (total; 51 patients). There was a significant relationship between groups. Thus with an increase in the levels of $\mathrm{iPTH}$, the prevalence of osteoporosis increased significantly $(P=0.03, r=0.035)$.

Based on the lumbosacral and femoral neck $\mathrm{T}$ scores, no significant relationship between iPTH levels and bone density was seen. In patients with iPTH $<100 \rho g / m L$, the total femur $\mathrm{Z}$ score in eight patients was more than (-2) and in five patients was less than (-2). In patients with $\mathrm{iPTH}=100-450 \rho \mathrm{g} / \mathrm{mL}$, the $\mathrm{Z}$ score was more than $(-2)$ in six patients and less than $(-2)$ in 15 patients. In patients with iPTH $>450 \mathrm{\rho g} / \mathrm{mL}$, the $\mathrm{Z}$ score was more than $(-2)$ in 12 patients and less than $(-2)$ in 39 patients. There was a significant association between the groups. Therefore, with an increase in the levels of $\mathrm{iPTH}$, the prevalence of osteoporosis increased significantly $(P=0.037, r=0.029)$. In patients with $\mathrm{iPTH}<100 \rho \mathrm{g} / \mathrm{mL}$, the femoral neck $\mathrm{Z}$ score was more than $(-2)$ in 12 patients and less than $(-2)$ in one patient. In patients with iPTH $=100-450 \mathrm{pg} /$ $\mathrm{mL}$, the $\mathrm{Z}$ score was more than $(-2)$ in 13 patients and less than $(-2)$ in six patients. In patients with iPTH $>450 \mathrm{pg} /$ $\mathrm{mL}$, the $\mathrm{Z}$ score was more than $(-2)$ in 25 patients and less than (-2) in 25 patients. Similarly a significant association between the groups was detected. While, with an increase in levels of iPTH, the prevalence of osteoporosis increased significantly $(P=0.008, r=0.015)$.

Based on the results of lumbosacral $Z$ scores, in patients with $\mathrm{iPTH}<100 \mathrm{\rho g} / \mathrm{mL}$, the $\mathrm{Z}$ score was more than $(-2)$ in 13 patients while in none of the patients it was less than $(-2)$. In patients with $\mathrm{iPTH}=100-450 \mathrm{gg} / \mathrm{mL}$, the $\mathrm{Z}$ score was more than $(-2)$ in 18 patients and less than $(-2)$ in one patient. In patients with iPTH $>450 \mathrm{\rho g} / \mathrm{mL}$, the Z score was more than $(-2)$ in 40 patients and less than $(-2)$ in 10 patients. Likewise, a significant association between the groups was seen. While, with an increase in the levels of $\mathrm{iPTH}$, the prevalence of osteoporosis increased significantly $(P=0.034, r=0.083)$ (Tables 4,5 and 6$)$.

To calculate the sensitivity and specificity of iPTH compared with patients' densitometry, the patients divided into two groups based on the T scores; a T score less than or equal to 2.5 and a $\mathrm{T}$ score more than 2.5. In

Table 3. Mean and standard deviation of blood parameters based on the state of osteoporosis in the studied subjects

\begin{tabular}{llcccc}
\hline & & \multicolumn{2}{c}{ T score Total } \\
\cline { 3 - 6 } & & Normal & Osteopenia & Osteoporosis & Total \\
\hline & Ca & $8.72 \pm 1.3$ & $8.66 \pm 1.05$ & $8.19 \pm 1.05$ & $8.00 \pm 1.03$ \\
& Vitamin D3 & $37.47 \pm 17.46$ & $29.6 \pm 26.3$ & $30.12 \pm 18.48$ & $29.92 \pm 21.55$ \\
& P & $5.23 \pm 1.16$ & $5.92 \pm 1.94$ & $5.89 \pm 1.71$ & $5.9 \pm 1.8$ \\
& Alp & $272.75 \pm 122.58$ & $454.16 \pm 405.49$ & $652.26 \pm 735.41$ & $547.96 \pm 604.31$ \\
Blood parameter & Mg & $2.42 \pm 0.68$ & $2.56 \pm 0.59$ & $2.73 \pm 0.61$ & $2.65 \pm 0.60$ \\
Mean \pm SD (n) & Alb & $3.87 \pm 0.41$ & $4.09 \pm 0.47$ & $3.96 \pm 0.43$ & $4.03 \pm 0.47$ \\
& FSH & $11.95 \pm 11.19(2)$ & $77.77 \pm 78.45(20)$ & $81.72 \pm 70.99(16)$ & $77.21 \pm 69.4(38)$ \\
& Testosterone & $3.66 \pm 0.62(2)$ & $3.22 \pm 1.86(11)$ & $3.43 \pm 1.6(27)$ & $3.39 \pm 1.62(40)$ \\
\cline { 2 - 6 } & T score spine & \multicolumn{4}{c}{} \\
\cline { 2 - 6 } & FSH & $61.23 \pm 71.08(11)$ & $82.36 \pm 64.39(21)$ & $80.63 \pm 78.22(6)$ & $77.21 \pm 69.4(38)$ \\
& Testosterone & $2.83 \pm 1.82(15)$ & $3.67 \pm 1.47(23)$ & $4.27 \pm 0.17(2)$ & $3.39 \pm 1.62(40)$ \\
\hline
\end{tabular}

There was no statistically significant difference between the groups. 
Table 4. Mean and standard deviation of PTH levels based on the state of osteoporosis in the studied subjects

\begin{tabular}{ccccc}
\hline & & Normal & Osteopenia & Osteoporosis \\
\hline \multirow{2}{*}{ Studied area } & Neck & $132.6 \pm 108.7$ & $402.41 \pm 395.42$ & $519.58 \pm 384.73$ \\
Mean \pm SD & Spine & $438.76 \pm 400.87$ & $472.11 \pm 328.62$ & $770.81 \pm 545.9$ \\
& Total & $211.05 \pm 198.36$ & $471.18 \pm 381.85$ & $532.47 \pm 395.45$ \\
\hline
\end{tabular}

There was no statistically significant difference between the groups.

Table 5. Mean and standard deviation of iPTH levels based on the state of osteoporosis by sex and age in the studied subjects

\begin{tabular}{|c|c|c|c|c|c|}
\hline & & Normal & Osteopenia & Osteoporosis & Total \\
\hline \multicolumn{6}{|c|}{ T score spins } \\
\hline & Male (\%) & $351.2 \pm 331.49$ & $396.93 \pm 314.83$ & $508.45 \pm 327.17$ & $385.35 \pm 315.29$ \\
\hline \multirow[t]{3}{*}{ Gender } & Female (\%) & $558.15 \pm 469.82$ & $554.46 \pm 330.95$ & $858.26 \pm 599.25$ & $610.18 \pm 429.84$ \\
\hline & Total (\%) & $438.76 \pm 400.87$ & $472.11 \pm 328.62$ & $770.81 \pm 545.9$ & $497.77 \pm 391.26$ \\
\hline & $<45$ & $377.4 \pm 148.1(3)$ & $602.81 \pm 349.09(14)$ & $333.05 \pm 266.5(2)$ & $538.82 \pm 320.5(24)$ \\
\hline Age group & $45-55$ & $562.74 \pm 512.96(12)$ & $495.18 \pm 408.32$ (9) & $1239.9 \pm 707.24(2)$ & $622.26 \pm 5013.6(23)$ \\
\hline \multirow[t]{2}{*}{ Mean $\pm S D(n)$} & $56-65$ & $400 \pm 346.05(2)$ & $348.35 \pm 285.33(15)$ & $-- \pm--(1)$ & $343.77 \pm 267.05(18)$ \\
\hline & $>65$ & $302.51 \pm 272.39(9)$ & $441.95 \pm 146.04(6)$ & $914.5 \pm 477.57(3)$ & $450.98 \pm 342.98(18)$ \\
\hline \multicolumn{6}{|c|}{ T score total } \\
\hline \multirow{3}{*}{ Gender } & Male (\%) & $124.55 \pm 120.13$ & $246.17 \pm 204.82$ & $461.38 \pm 336.14$ & $385.35 \pm 315.29$ \\
\hline & Female (\%) & $297.55 \pm 271.45$ & $594.93 \pm 403.38$ & $652.44 \pm 466.45$ & $610.18 \pm 429.84$ \\
\hline & Total (\%) & $211.05 \pm 198.36$ & $471.18 \pm 381.85$ & $532.47 \pm 395.45$ & $497.77 \pm 391.26$ \\
\hline \multirow{4}{*}{$\begin{array}{l}\text { Age group } \\
\text { Mean } \pm S D(n)\end{array}$} & $<45$ & $349.5 \pm 197.98(2)$ & $572.87 \pm 338.39(9)$ & $547.85 \pm 340.37(8)$ & $538.82 \pm 320.5(19)$ \\
\hline & $45-55$ & - & $482.22 \pm 484.62(12)$ & $779.72 \pm 510.51(10)$ & $622.26 \pm 5013.6(23)$ \\
\hline & $56-65$ & - & $376.75 \pm 288.27(8)$ & $328.83 \pm 274.47(10)$ & $343.77 \pm 267.05$ (18) \\
\hline & $>65$ & - & $325.05 \pm 290.83(2)$ & $495.2 \pm 346.92(15)$ & $450.98 \pm 342.98(18)$ \\
\hline
\end{tabular}

In the osteoporosis group (spin T score) the difference was significant $(P<0.05)$, in the other cases there was no statistically significant difference between the groups.

Table 6. Relative and absolute frequency distribution of the studied subjects based on the state of osteoporosis (T score spin and total) and iPTH

\begin{tabular}{|c|c|c|c|c|c|}
\hline & & Normal & Osteopenia & Osteoporosis & Total \\
\hline \multirow{2}{*}{ Adynamic bone disease } & T score total, No. (\%) & $2(2.6)$ & $7(9)$ & $2(2.6)$ & $11(14.1)$ \\
\hline & T score spine, No. (\%) & $6(7.7)$ & $5(6.4)$ & $0(0)$ & $11(14.1)$ \\
\hline \multirow{2}{*}{ Normal } & T score total, No. (\%) & $1(1.3)$ & $6(7.7)$ & $13(16.7)$ & $20(25.6)$ \\
\hline & T score spine, No. (\%) & $7(9)$ & $12(15.4)$ & $1(1.3)$ & $20(25.6)$ \\
\hline \multirow{2}{*}{ Hyperparathyroidism } & T score total, No. (\%) & $1(1.3)$ & $18(23.1)$ & $28(35.9)$ & $47(60.3)$ \\
\hline & T score spine, No. (\%) & $13(16.7)$ & $27(34.6)$ & $7(9)$ & $47(60.3)$ \\
\hline \multirow{2}{*}{ Total } & T score total, No. (\%) & $4(5.1)$ & 31 (39.7) & $43(55.1)$ & $78(100)$ \\
\hline & T score spine, No. (\%) & $26(33.3)$ & $44(56.4)$ & $8(10.3)$ & $78(100)$ \\
\hline
\end{tabular}

There was no significant relationship between the state of osteoporosis and T score spin.

Considering $P \leq 0.05$, there was a statistically significant relationship between osteoporosis and T score total.

addition, the patients were also divided into two groups based on the $\mathrm{Z}$ scores; a $\mathrm{Z}$ score less than or equal to 2 and a $\mathrm{Z}$ score more than 2 . Patients were divided into three groups based on their iPTH: patients with iPTH $<100$ pg/ $\mathrm{mL}$, patients with $\mathrm{iPTH}=100-450 \rho \mathrm{g} / \mathrm{mL}$, and patients with $\mathrm{iPTH}>450 \mathrm{\rho g} / \mathrm{mL}$. In both groups, the low and high levels were compared with the normal group. In adynamic cases, the sensitivity and specificity of iPTH compared with lumbosacral $\mathrm{T}$ score was $0 \%$ and $95 \%$, respectively. In hyperparathyroidism cases, the sensitivity and specificity of iPTH compared with lumbosacral T score was $21 \%$ and $95 \%$, respectively. In adynamic cases, the sensitivity and specificity of iPTH compared with femoral neck T score was $19 \%$ and $46 \%$, respectively. In hyperparathyroidism cases, the sensitivity and specificity of iPTH compared with femoral neck T score was $19 \%$ and $82 \%$, respectively.
In adynamic cases, the sensitivity and specificity of iPTH compared with total femur $\mathrm{T}$ score was $33 \%$ and $15 \%$, respectively. In hyperparathyroidism cases, the sensitivity and specificity of iPTH compared with total femur T score was $30 \%$ and $60 \%$, respectively.

In adynamic cases, the sensitivity and specificity of iPTH compared with lumbosacral Z score was $0 \%$ and $94 \%$, respectively. In hyperparathyroidism cases, the sensitivity and specificity of iPTH compared with lumbosacral Z score was $94 \%$ and $30 \%$, respectively. In adynamic cases, the sensitivity and specificity of iPTH compared with femoral neck $\mathrm{Z}$ score was $68 \%$ and $7 \%$, respectively. In hyperparathyroidism cases, the sensitivity and specificity of iPTH compared with femoral neck $\mathrm{Z}$ score was $68 \%$ and $50 \%$, respectively. In adynamic cases, the sensitivity and specificity of iPTH compared with total femur Z score 
was $28 \%$ and $38 \%$, respectively. In hyperparathyroidism cases, the sensitivity and specificity of iPTH compared with total femur $\mathrm{Z}$ score was $28 \%$ and $68 \%$, respectively.

\section{Discussion}

End-stage renal failure is a costly and debilitating disease, which its incidence has decreased in recent decades. Nowadays, there is limited data available about the effects of hemodialysis on bone density in patients with end-stage renal failure. Most of the available studies are conducted on pre-dialysis patients and kidney transplant patients; the studies conducted on dialysis patients usually have assessed the factors which are involved in the reduction of bone density in dialysis patients and they seldom have specifically investigated the relationship between parathormone and bone density. On the other hand, in most studies densitometry is limited to specific areas and they rarely use densitometry tests for various part of the body. Given that different parts of the body have different bone structure which can be affected by numerous factors, it is necessary to examine various parts of the body. In this study, we evaluated the lumbosacral, total femur, and femoral neck areas of the body.

The prevalence of end-stage renal failure, which is known as the fifth stage of CKD in the United States of America, is equal to $0.2 \%$ of the total population of this country (3). The proportion of patients with chronic renal failure in Iran, according to the latest statistics published was 32,686 people. Of them, 15957 patients are treated by hemodialysis (4).

The prevalence of osteoporosis in hemodialysis patients is different and depends on various factors, including the method of dialysis, the place of measurement, and gender. However, most studies show a decrease in bone density in patients undergoing hemodialysis.

The prevalence of osteoporosis in lumbosacral region is reported to be $13 \%$ to $29 \%$, while in femoral neck region it is reported to be $16 \%$ to $20 \%$ (1). In our study, of all the 85 dialysis patients, $78.8 \%$ had osteoporosis and $17.6 \%$ had osteopenia in the femoral neck region, $54.1 \%$ had osteoporosis and $40 \%$ had osteopenia in the total femur region. Additionally, around $10.6 \%$ had osteoporosis and $57.6 \%$ had osteopenia in the lumbosacral region.

In the femoral neck region $37.6 \%$ had a $\mathrm{Z}$ score less than -2 and in the lumbosacral $10.6 \%$ had a $Z$ score less than -2 . These results suggest the need for further studies on the secondary causes.

In a cross sectional study by Huang et al at the University of Taiwan, various factors involved in the reduction of bone density in 63 patients with ESRD was evaluated. They found, 51 patients had a score less than $(-1)$ and eight patients (13\%) were osteoporotic. The results showed that femoral neck was more involved. They found, an inverse relationship between the old age, low level of albumin, high level of ALP, high level of PTH, and bone density in femoral neck and lumbar spine regions (7). In our study, a significant relationship between patients' age and bone density measured by the total femur $\mathrm{T}$ score, femoral neck $\mathrm{Z}$ score, and lumbosacral $\mathrm{Z}$ score was detected. Therefore, with increasing age, the bone density decreased and with increasing the level of PTH, the prevalence of osteoporosis increased significantly accordingly.

In a meta-analysis by Jamal et al, based on studies conducted between 2006-1999, the relationship between bone fractures and reduced levels of bone density was investigated. They showed bone density in patients with stage $5 \mathrm{CKD}$ led to fewer cases of bone fractures. The authors suggested that further investigation on the relationship can be helpful to determine the risk of fractures in the future. For example, in the five studies which had evaluated the relationship between bone density and vertebral fractures it was found that pooled standardized mean difference was - 0.44 (95\% CI: -0.800.08) (8).

In the cross-sectional study conducted on 70 patients at the University of Paris, Urena et al evaluated the relationship between bone density and biochemical markers and fractures in hemodialysis patients. They concluded that in patients undergoing hemodialysis, the $\mathrm{Z}$ score reduced in the mid-radius area and the decline was associated with an increased levels of PTH, while it was not associated with fractures [Mean Z score in mid-radius $(-2.75 \pm 1.25)$, femoral neck $(-0.42 \pm 1.13)$ and lumbar spine $(-0.02 \pm$ 2.13)] (9).

Furthermore, Marianne et al evaluated the relationship between Bone mineral density (BMD) and biochemical markers related to bone turn over in pre-dialysis CKD patients. The study was conducted on 128 patients at the University of Copenhagen, Denmark (1999). They concluded that skeletal changes occur in the early stages of CKD. In addition bone density was significantly reduced in the patients, compared with the control group [BMD spine $(-6.3 \%)$, femur $(12.1 \%)$, forearm $(-5.7 \%)$, total body (-4.2\%)]. Furthermore, a relationship between bone density and the increase in PTH levels and biochemical markers which are involved in bone resorption and formation of bone was seen $(P<0.05$ to $P<0.001)(10)$.

Moreover, the study conducted by Gupta et al at the University of Kuwait showed that the hemodialysis patients had lower BMD compared with the control group [BMD hip (0.81 \pm 0.11 versus $0.92 \pm 0.16)$, spine (0.84 \pm 0.12 vs $0.92 \pm 0.16), P<0.001]$. In addition, the risk of fracture was more in hemodialysis patients (11).

In addition, in the study by Ambrus et al (12), which was conducted at the University of Koranyia, the relationship between BMD and parathyroid performance in hemodialysis patients was examined. The results showed no significant difference between the mean $\mathrm{Z}$ scores of the group with low PTH (iPTH $<100 \rho g / m L)$ compared with the group at the target iPTH level $(100-300 \mathrm{\rho g} / \mathrm{mL})$. The relationship between BMD and $\mathrm{PTH}$ in patients with $\mathrm{PPTH}$ $>100 \mathrm{\rho g} / \mathrm{mL}$ was negative ( $\mathrm{rho}=-0.255,-0.275,-0.251$ for LS, FN and DR, $P<0.001)$. However in patients with iPTH $<100 \mathrm{\rho g} / \mathrm{mL}$, was no relationship between PTH and BMD 
was found. In addition, no relationship between markers of bone metabolism and PTH was detected. However, in low iPTH group these markers were negatively correlated with BMD (12).

In fact, numerous studies mentioned a relationship between the increases of PTH with the decrease in bone density in hemodialysis was mentioned. In our study, concerning the iPTH level, $15.3 \%$ of patients were placed in the adynamic group, $24.7 \%$ in the normal group, and $60 \%$ in the hyperparathyroidism group. We found, a significant relationship between the groups regarding of total femur $\mathrm{T}$ score, total femur $\mathrm{Z}$ score, femoral neck $\mathrm{Z}$ score, and lumbosacral $\mathrm{Z}$ score, while with increasing the levels of iPTH, the bone density decreased.

In our analysis, a significant relationship between age and bone density regarding of total femur $T$ score, femoral neck $\mathrm{Z}$ score, and lumbosacral Z score was seen. Hence, with increasing age, the bone density decreases. In our study, we assessed the sensitivity and specificity of PTH and we compared it with the results of densitometry tests. The results showed that in patients with iPTH $>450 \mathrm{\rho g} / \mathrm{mL}$ the sensitivity and specificity were $82 \%$ and $19 \%$, respectively. For femoral neck $\mathrm{T}$ score it was $60 \%$ and finally $30 \%$ for total femur $\mathrm{Z}$ score. In other cases the sensitivity of the test was low.

\section{Conclusion}

Finally it was concluded that the prevalence of osteopenia and osteoporosis in hemodialysis patients is high and is associated with high levels of PTH. As they are mainly associated with $\mathrm{Z}$ score, it seems necessary to investigate the secondary causes of this condition.

\section{Limitations of the study}

This investigation was conducted on a limited proportion of patients. Thus, larger studies on this feature of hemodialysis patients are necessary.

\section{Authors' contribution}

All authors passed four criteria for authorship contribution based on recommendations of the International Committee of Medical Journal Editors. Study concept and design: BO and AG. Acquisition of data: ZSB and MD. Analysis and interpretation of data: MB. Drafting of the manuscript: MRT and MB. Critical revision of the manuscript for important intellectual content: AG. Statistical analysis: MB. Administrative, technical, and material support: MRT and MB. Study supervision: AG.
Funding/Support

This study was extracted from internal medicine residency thesis of Zahra Sadeghian Broujeni (U-90188), in Ahvaz Jundishapur University of Medical Sciences, Ahvaz, Iran.

\section{References}

1. Mousavi SS, Soleimani A, Mousavi MB. Epidemiology of end-stage renal disease in Iran: a review article. Saudi J Kidney Dis Transpl. 2014;25:697-702.

2. Collins AJ, Foley RN, Chavers B, Gilbertson D, Herzog C, Johansen K, et al. 'United States Renal Data System 2011 Annual Data Report: Atlas of chronic kidney disease \& end-stage renal disease in the United States. Am J Kidney Dis. 2012;59:A7, e1-420. doi: 10.1053/j.ajkd.2011.11.015.

3. Coresh J, Byrd-Holt D, Astor BC, Briggs JP, Eggers PW, Lacher DA, et al. Chronic kidney disease awareness, prevalence, and trends among U.S. adults, 1999 to 2000. J Am Soc Nephrol. 2005;16:180-8.

4. Jon J. Snyder, Robert N. Foley, Allan J. Collins Prevalence of CKD in the United States: A Sensitivity Analysis Using the National Health and Nutrition Examination Survey (NHANES) 1999-2004. Am J Kidney Dis. 2009;53:218-228.

5. Sanusi AA, Arogundade FA, OladigboM, Ogini LM, Akinsola A. Prevalence and pattern of renal bone disease in end stage renal disease patients in Ile-Ife, Nigeria. West Afr J Med. 2010;29:75-80.

6. Raisz LG. Clinical practice. Screening for osteoporosis. N Engl J Med. 2005;353:164-71.

7. Huang GS, Chu TS, Lou MF, Hwang SL, Yang RS. Factors associated with low bone mass in the hemodialysis patients-a cross-sectional correlation study. BMC Musculoskelet Disord. 2009;10:60.

8. Jamal SA, Hayden JA, Beyene J. Low bone mineral density and fractures in long-term hemodialysis patients: a metaanalysis. Am J Kidney Dis. 2007;49:674-81.

9. Urena P, Bernard-Poenaru O, Ostertag A, Baudoin C, Cohen-Solal M, Cantor T, et al. Bone mineral density, biochemical markers and skeletal fractures in haemodialysis patients. Nephrol Dial Transplant. 2003;18:2325-31.

10. Rix M, Andreassen H, Eskildsen P, Langdahl B, Olgaard $\mathrm{K}$. Bone mineral density and biochemical markers of bone turnover in patients with predialysis chronic renal failure. Kidney Int. 1999;56:1084-93.

11. Gupta R, Mohammed AM, Alenizi EK, Ben Nekhi A. Bone mineral density in Kuwaiti patients with end-stage renal disease. Med Princ Pract. 2011;20:156-8.

12. Ambrus C, Almasi C, Berta K, Deak G, Marton A, Molnar $\mathrm{MZ}$, et al. Bone mineral density and parathyroid function in patients on maintenance hemodialysis. Int Urol Nephrol. 2011;43:191-201.

\section{Conflicts of interest}

The authors declare no conflict of interest. 